\title{
DESIGN AND DEVELOPMENT OF ADJUSTABLE STAIR CLIMBING ROBOT
}

\author{
K. Narendra Kumar', A. Gopichand ${ }^{2}$, M. Gopala Anjaneyulu ${ }^{3}$, B. Gopi Krishna ${ }^{4}$ \\ ${ }^{1}$ Assistant Professor, ${ }^{2}$ Associate Professor, ${ }^{3,4}$ Student, ${ }^{1,2,3,4}$ Department of Mechanical Engineering, Swarnandhra \\ College of Engineering and Technology, Narsapur, Andhra Pradesh, India- 534275 \\ narendra.or.kumar@gmail.com, allakagopichand@gmail.com,gopala.anji@gmail.com,bgk303@gmail.com
}

\begin{abstract}
In the present scenario the application of robots is quite common to reduce the human effort in several areas. The stair climbing robots are used to climb the stairs for different applications up to now, but the main disadvantage of the rugged terrain robots is not adjustable according to the structure of the stairs. To overcome this, we have developed an adjustable stair climbing robot to climb the stairs up and down according to the dimensions of the staircase by using adjustable frame. The main features of the robot include the platform which is attached to the adjustable frame to carry the materials up and down as per the motor capacity. The main application of this robot is to check the urban areas by carrying the materials on the platform for rescue operations.
\end{abstract}

Keywords: Adjustable frame, Staircase, circuit board, Design, Robot Wheels.

\section{INTRODUCTION}

Adjustable stair climbing robot is one of the most attractive performances of robot in legged and wheeled. Developments have been made on various kinds of stair climbers, considering how to make its climbing ability higher and its mechanical complexity reasonable and practical. The research includes realizing a large step negotiating. Reducing body weight and energy consumption is also the important matter of developing. We introduce some solutions to realize stair climbing machines that we developed. Each of them has good performance as in a category of their kind, e.g. various numbers of wheeled shapes. Then, we discuss a development of adjustable high-grip mover, which we think one of the best solutions as the stair climber.A mechanism is a combination of rigid or restraining bodies so shaped and connected that they move upon each other with definite relative motion. A machine is a collection of mechanisms which transmits force from the source of power to the load to be overcome, and thus perform useful mechanical work. Robotics is the area of automation which integrates the technology in variegated fields like mechanisms, sensors \& electronic control systems, artificial intelligence and embedded systems. The synthesis of mechanisms is the very first step in any robot design depending upon its application.

\section{RELATED WORK}

Stair climbing has been carried out with robots using different types of mechanisms. One can roughly distinguish wheeled, legged, and tracked robots.

\subsection{WHEELED ROBOTS}

Wheeled robots usually have to resort to mechanic extensions to overcome stairs. One application of such a technique is inpatient rehabilitation, where stair climbing could greatly enhance mobility, and thus quality of life, of people confined to wheelchairs. Lawn and Shiatsu [1] present a stair-climbing wheelchair using two (forward and rear) articulated wheel clusters attached to movable appendages. The robot is equipped with step-contact sensors, but relies on user steering and is thus only semi-autonomous.
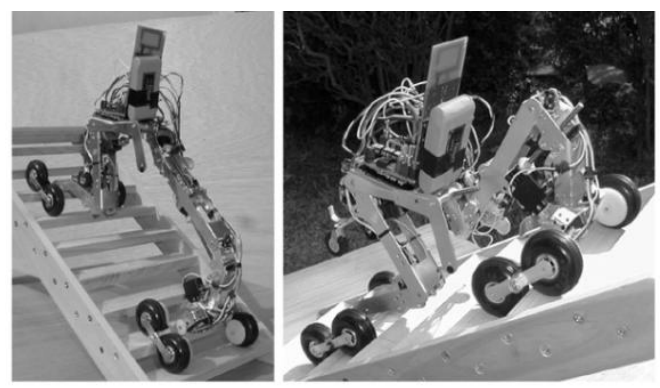

Fig-1: Wheeled Robots

\subsection{LEGGED ROBOTS}

Figliolini and Ceccarelli[2] present the architecture of the bipedal robot EP-WAR2, that uses electro pneumatic actuators and suction cups for locomotion. In order to climb stairs, the robot relies on an open-loop control algorithm implemented as a finite-state machine. The main limitation of the approach is 
that operating in a different staircase necessitates manual recalibration

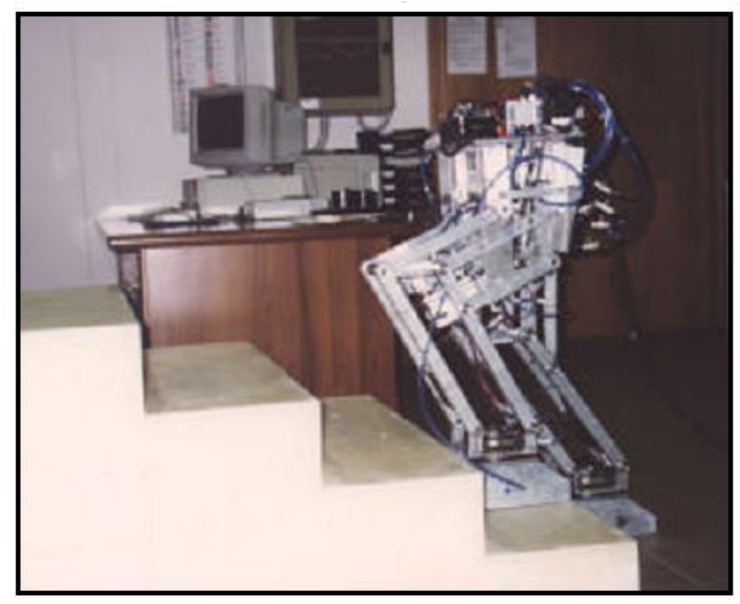

Fig-2: Legged Robots

\subsection{TRACKED ROBOTS}

Tracked robots have a larger ground contact surface than wheeled vehicles and are more stable than bipeds due to their low center of gravity. Liu et al.[3] derived the fundamental dynamics of the stair-climbing process for a tracked robotic element, analyzing the different phases of riser climbing, nose crossing, nose line climbing and the effects of grouser bars or cleats. The analysis is limited to $2 \mathrm{D}$, and slippage, shocks, and intermittent loss of track-surface contact, phenomena that are commonly encountered during stair climbing, are neglected.

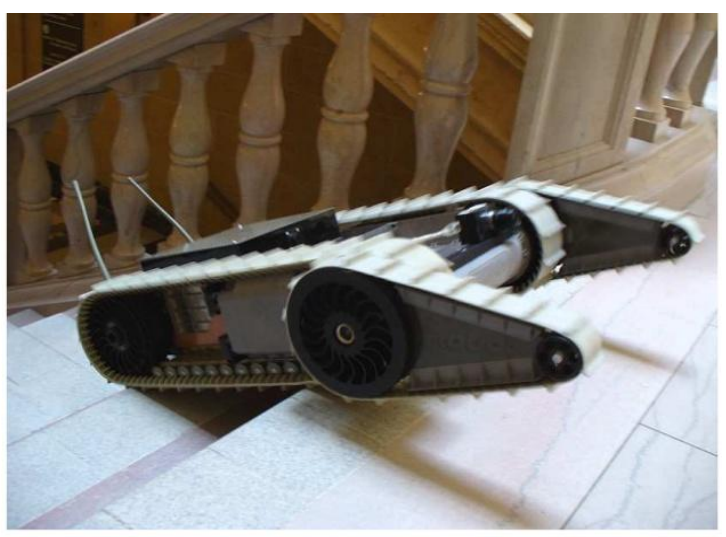

Fig-3: Tracked Robots

\section{DESIGN OF ROBOT}

In the present work we have designed the robot parts and assembled the parts using Pro-E Wildfire.

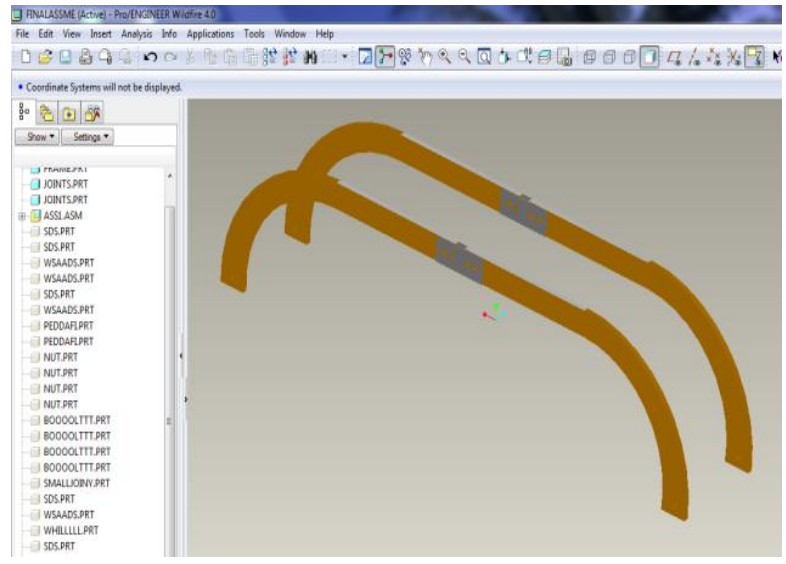

Fig-4: Design model of adjustable frame

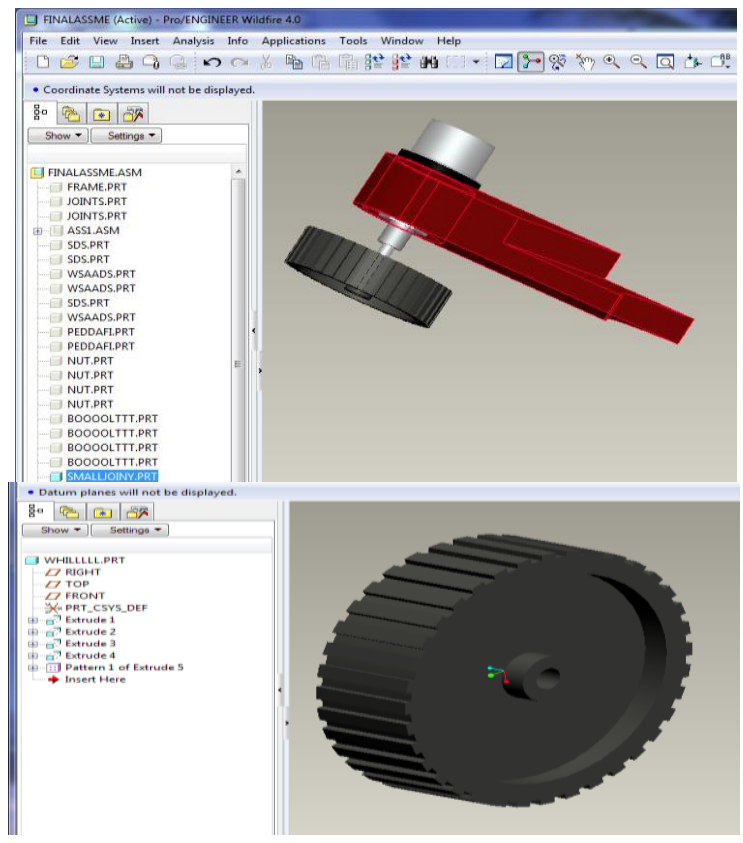

Fig-5: Design model of upper wheel

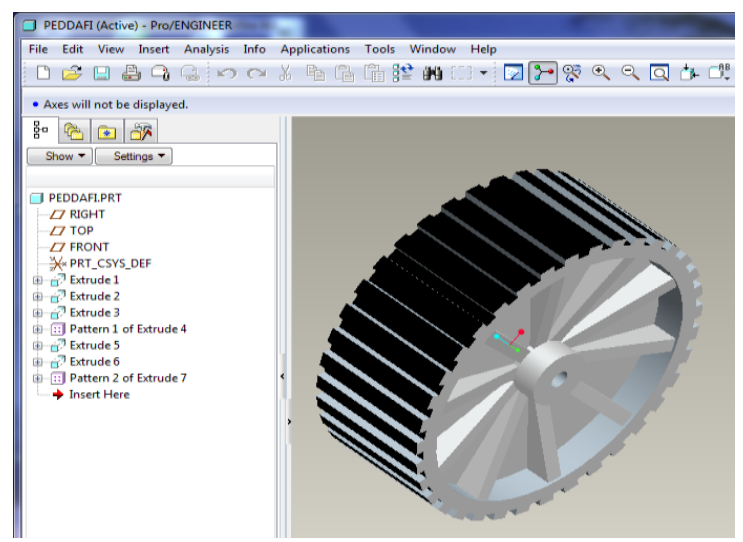

Fig-6: Design model of lower wheel 


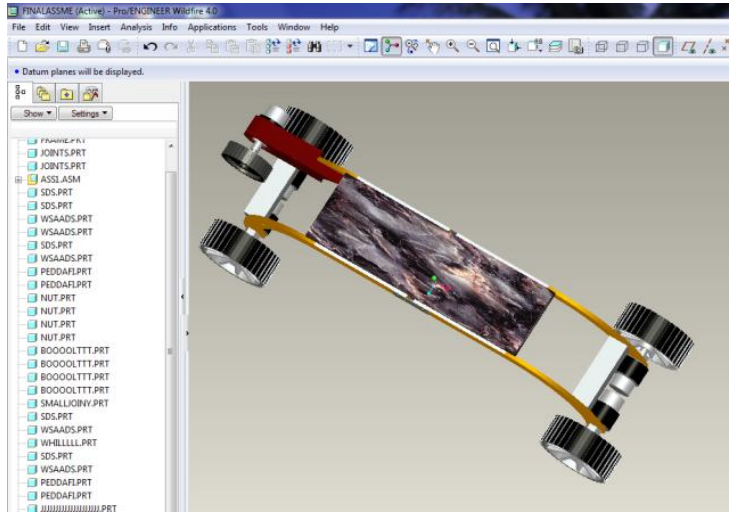

Fig-7: Assembly of Adjustable stair climbing robot

\section{MECHANISM OF A ROBOT}

The adjustable stair climbing robot consists of a adjustable wooden frame, D.C induction motors, Wheels, On-Off Switch, Connecting wires, circuit board and a platform.

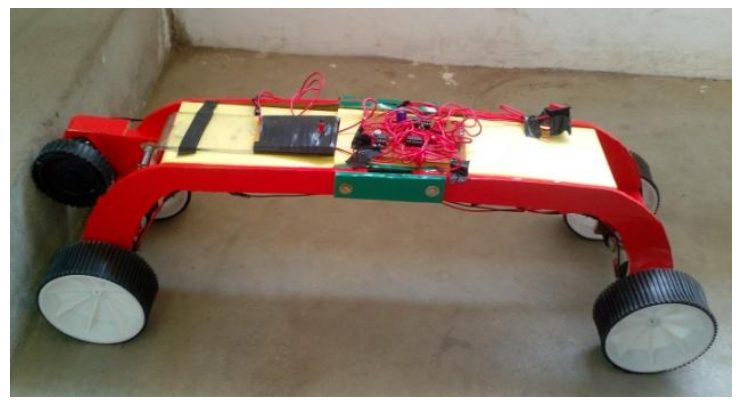

Fig-8: Adjustable Stair Climbed Robot

\subsection{DESCRIPTION OF PARTS:}

\subsubsection{AdJUSTABLE FrAME:}

The main feature of this robot is adjustable wooden frame. We can adjust the length of the robot with respect to stair size.

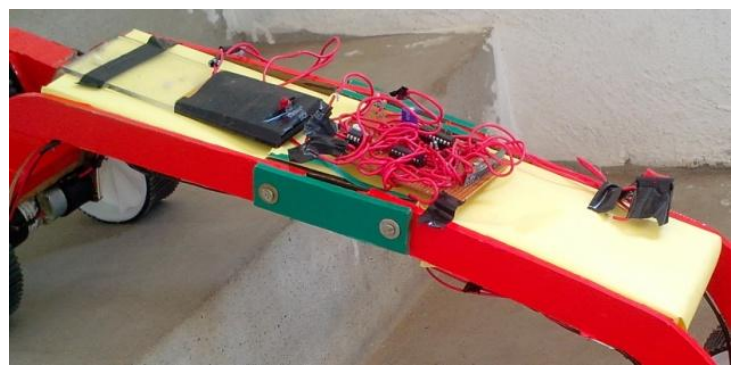

Fig-9: Adjustable Frame of the Robot

\subsubsection{PlATFORM}

This platform is used to carry the materials up and down. It is made by wooden board attached to the frame.

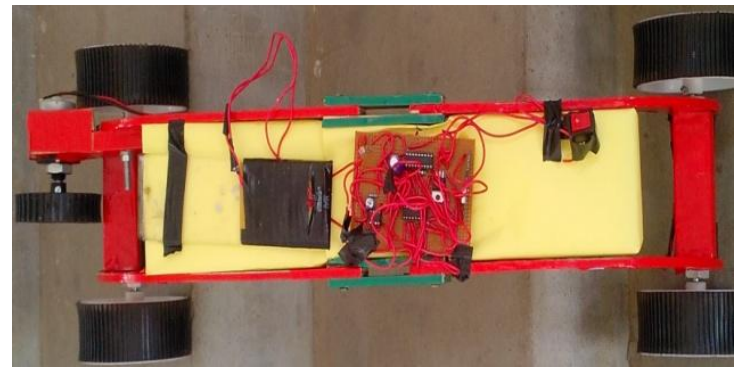

Fig-10: Platform attached to the Frame

\subsubsection{CIRCUIT BOARD}

We can move the robot forward and backward with the help of remote control. The circuit board is designed for moving the robot which can climbs the stairs up and down. It is a wireless circuit which produces $12 \mathrm{v}$ when moving up and $6 \mathrm{v}$ when moving down.

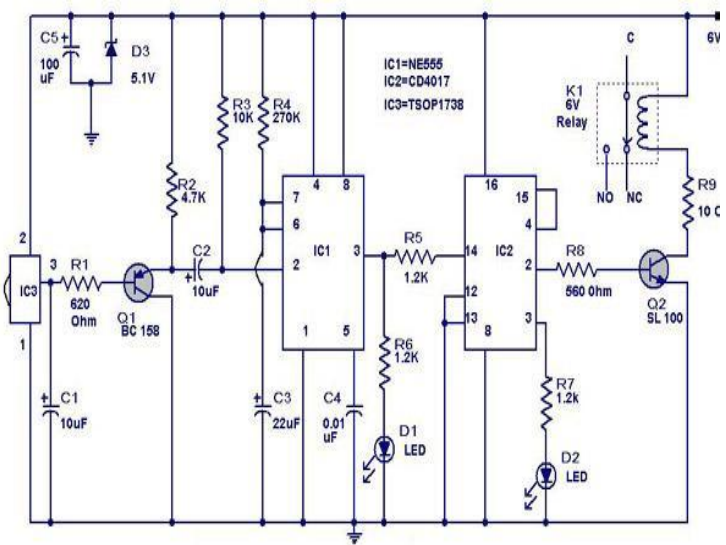

Fig-11: Block Diagram of Circuit board

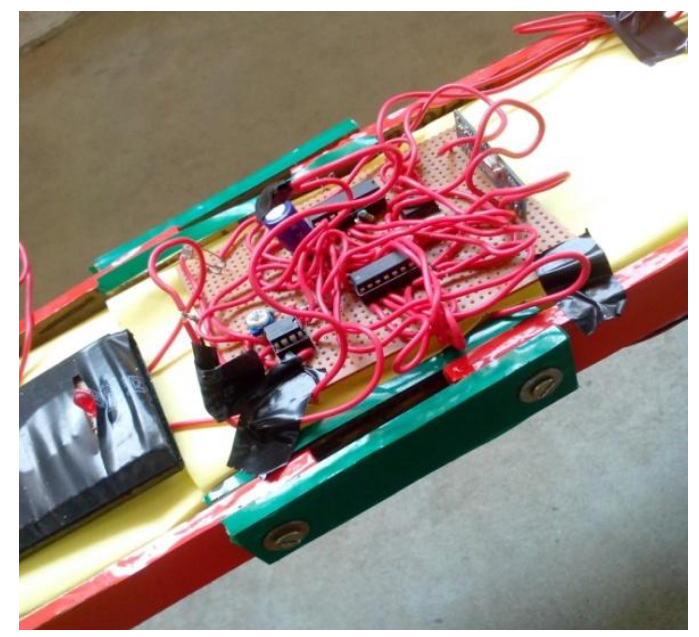

Fig-12: Circuit board placed on Platform 


\subsection{PARTS SPECIFICATIONS:}

Table 1: Robot parts specifications

\begin{tabular}{|c|c|c|}
\hline Part & Specification & Quantity \\
\hline Motor & $\begin{array}{l}\text { 12v DC Gear Motor } \\
60 \text { RPM }\end{array}$ & 5 \\
\hline Upper wheel & $7 \mathrm{~cm} \times 2 \mathrm{~cm}$ & 1 \\
\hline Lower wheels & $10.5 \mathrm{cmx} 4.2 \mathrm{~cm}$ & 4 \\
\hline Battery & $12 \mathrm{v}, 1500 \mathrm{mah}$ & 1 \\
\hline Circuit Board & $\begin{array}{l}12 \mathrm{v}-\text { moving up and } \\
6 \mathrm{v}-\text { moving down. }\end{array}$ & 1 \\
\hline $\begin{array}{l}\text { Adjustable } \\
\text { frame }\end{array}$ & $\begin{array}{l}\text { Length can be } \\
\text { adjust able }\end{array}$ & 1 \\
\hline Platform & $420 \mathrm{~mm} \times 102 \mathrm{~mm}$ & 1 \\
\hline
\end{tabular}

\subsection{STEPS FOR MAKING THE ROBOT:}

$>$ First design the wooden frame as per the dimensions with adjustable mechanism.

$>$ The structure of the robot should be rigid.

$>$ The five D.C motors are attached to the end of the frame.

$>$ Four $12 \mathrm{v}$ batteries are connected in series for constant movement of wheels.

$>$ The positive connection is upto12v, and negative connection is up to $6 \mathrm{v}$.

$>$ Positive connection is for forward motion and negative connection is for backward motion.

$>$ These connections are linked to circuit board for the robot motion.

$>$ The robot can be controlled by remote which is connected to circuit.

$>$ The remote has a switch which is helpful for the backward and forward motion of robot.

\subsection{WORKING OF THE ROBOT}

Working of the robot takes place stepwise. The robot comes to rest momentarily after each step. The four steps for climbing the stairs are

1. Robot wheel touches the step

2. Lifting the front part.

3. Lifting the back part of the robot.

4. Following the above steps the robot proceeds.

5. It can also be used for descending of steps.

\subsubsection{ROBOT WHEEL TOUCHES THE STEP}

Initially the robot is in horizontal position and when the robot touches the first step, the upper wheel is ready to move upward to lift the front part of the robot.

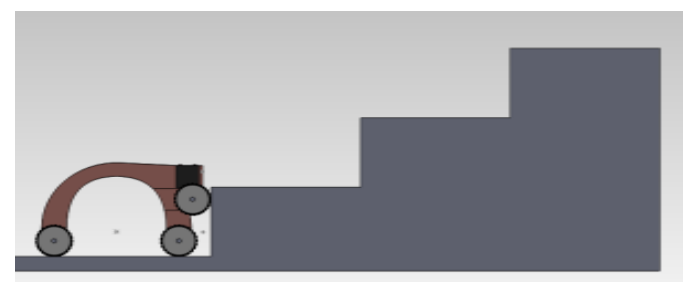

Fig-13: Robot is in initial position

\subsubsection{LIFTING THE FRONT PART}

By switching on, the motor starts rotating the upper wheel which lifts the front part of the robot to a certain height. The front wheels will move forward through the step and also helps the robot to climb the stair.

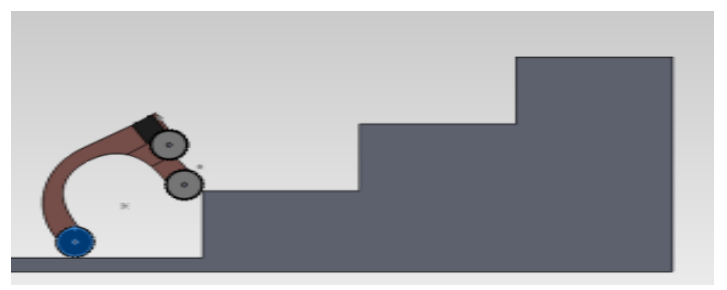

Fig-14: Lifting the front part.

\subsubsection{LIFTING THE BACK PART OF THE ROBOT}

After lifting the front part of the robot, the rear wheel touches the step and moves forward. After climbing all the steps, it will reaches to its initial position.

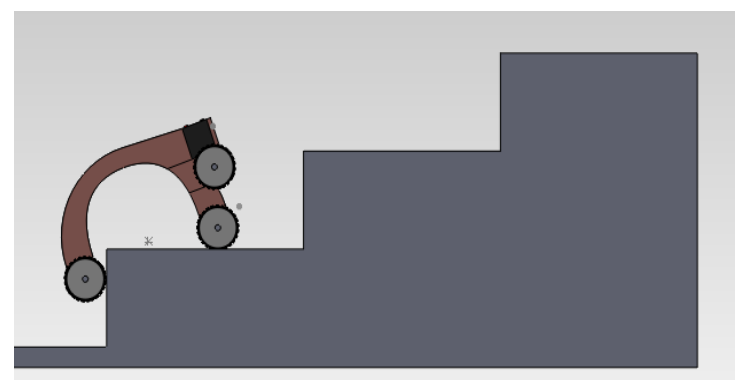

Fig-15: Lifting the rear part of Robot

\section{EXPERIMENTAL RESULTS}

We are succeeded to run the stair climbed robot to climb the stairs up and down which has shown in figures displayed below. 


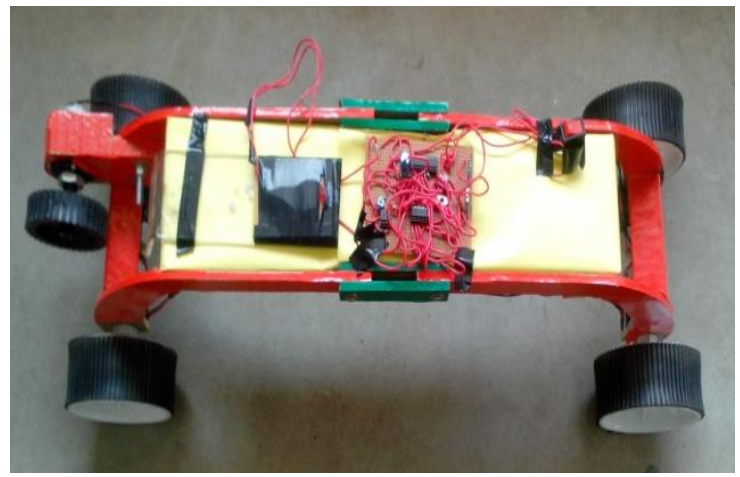

Fig-16: Robot is in initial position

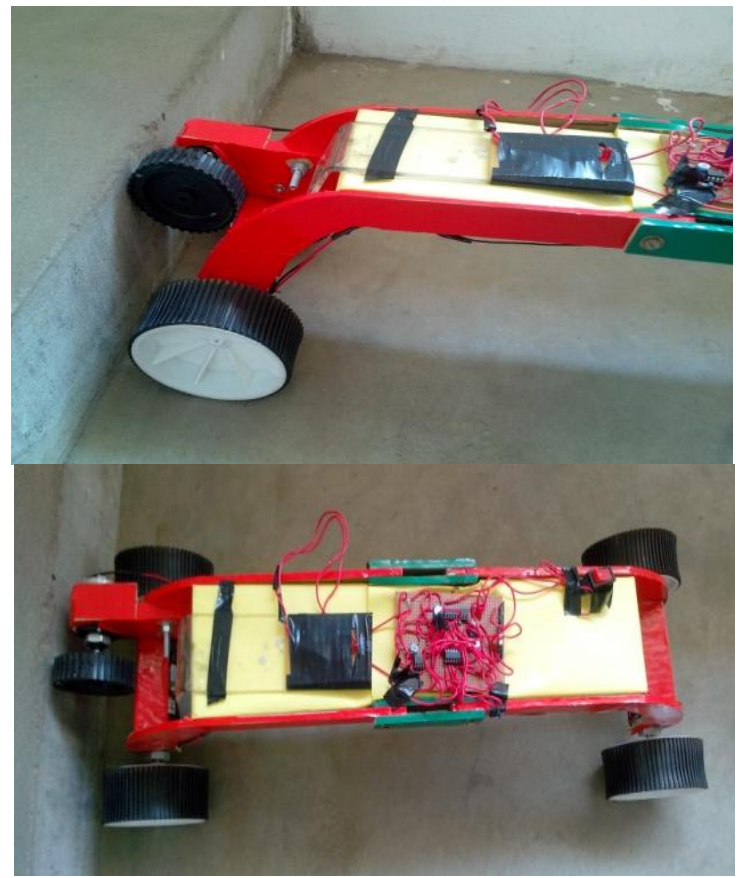

Fig-17: The front and top view of the robot which touches the step

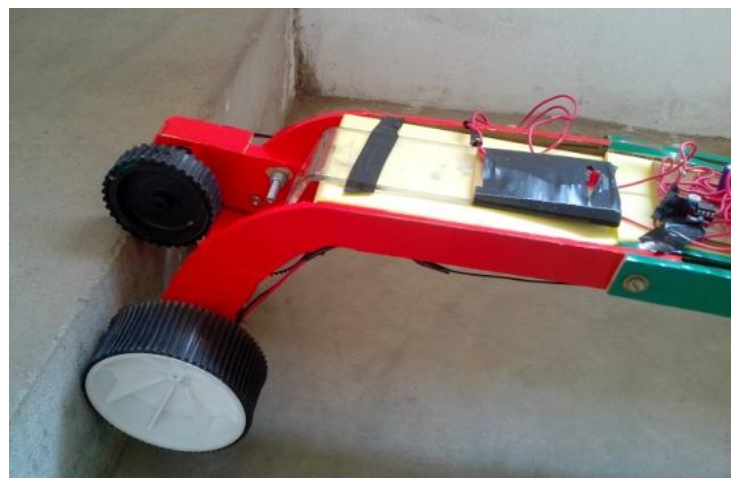

Fig-18: The Upper Wheel lifts the Front part of the Robot

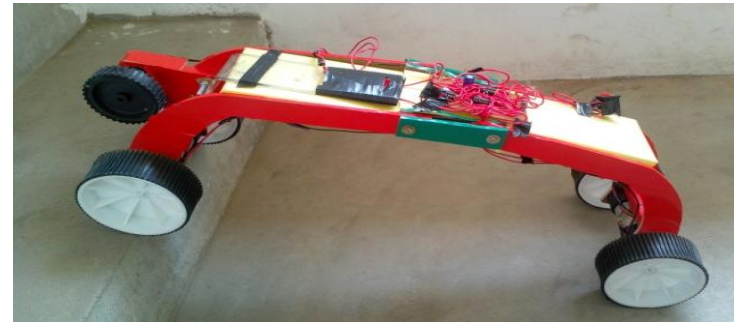

Fig-19: The Front wheels which climbs the step up

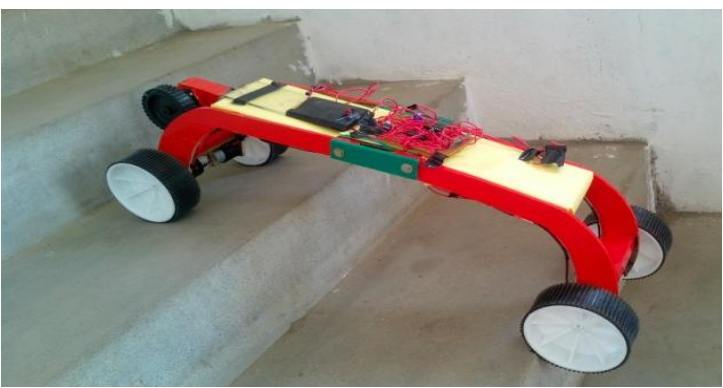

Fig-20: The Rear wheels which climbs the step up

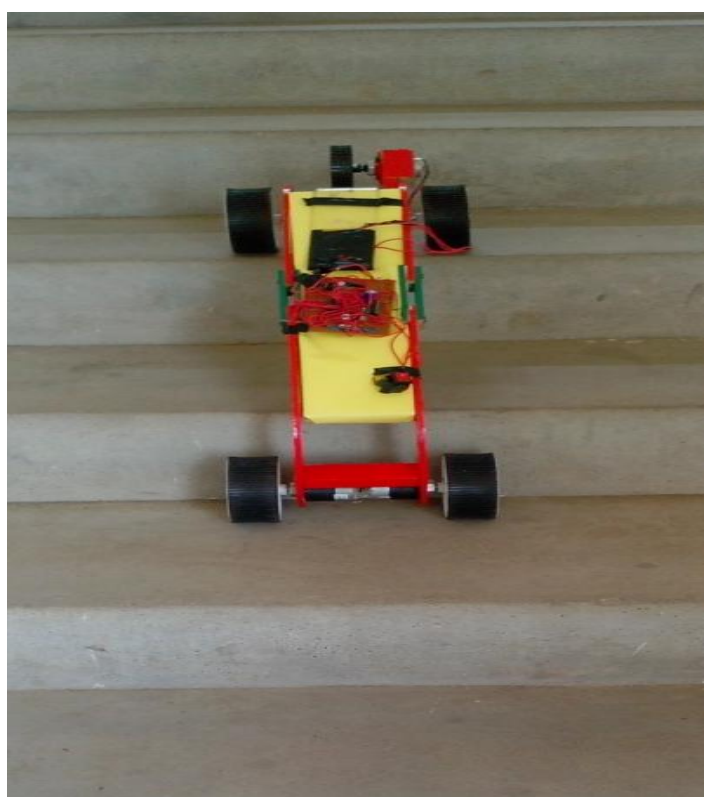

Fig-21: Robot climbing stairs up and down

\section{CONCLUSION AND FUTURE SCOPE}

In this paper we have developed Adjustable stair climbing robot for providing the service to replace the human in many fields like office, military tasks, hospital operations, industrial automation, security systems and dangerous environment. The developed model has completed the task successfully.

This mechanism can be further modified and used in various other applications. It can be useful in carrying heavy baggage or load and thus reducing human effort. Another common 
situation that employs the use of this mechanism is in the case of urban disasters or hostage situations wherein these robots are designed to rescue workers. The benefits of rescue robots to these operations include personnel requirements, reduced fatigue as well as access to unreachable areas. These robots have the ability to move over irregular terrain of collapsed or destroyed buildings. On the robot, a camera can be placed to take a video of the affected areas which can further help in rescuing operations.

\section{REFERENCES:}

[1] M. Lawn and T. Shiatsu, "Modeling of a stair-climbing wheelchair mechanism with high single-step capability," IEEE Transactions on Neural Systems and Rehabilitation Engineering, vol. 11, no. 3, pp. 323-332, Sept. 2003

[2] G. Figliolini and M. Ceccarelli, "Climbing stairs with EPWAR2 biped robot," in Proc. IEEE International Conference on Robotics and Automation (ICRA), vol. 4, Seoul, Korea, 2001, pp. 4116-4121

[3] J. Liu, Y. Wang, S. Ma, and B. Li, "Analysis of stairsclimbing ability for a tracked reconfigurable modular robot," in Proc. IEEE International Workshop on Safety, Security and Rescue Robotics, Kobe, Japan, June2005, pp. 53-38

[4] R. C. Luo, K. L. Su, "A multi agent multi sensor based real-time sensory control system for intelligent security robot" IEEE International Conference on Robotics and Automation, vol. 2, 2003, pp.2394-2399.

[5] Sandeep H. Deshmukh, Sakthivel P. \& Srikanth Sankaran, "Computer Aided Design and Interfacing Of EOT Crane" in the Proc. of Global Conference on Production and Industrial Engineering ,National Institute of Technology, Jalandhar, 2007, Session 4A, pp 1-6

[6] Stoeter et al., 2002; Murphy, 2000; Yim et al., 2000; Krishna et al.,1997; Granosik et al., 2005; Liu et al., 2005; Arai et al., 2006; Tanaka et al., 2006; Miyanaka et al.,2007; Tsukagoshi et al.,2005

[7] "Algorithmic Foundations of Robotics VI" By Michael Erdmann, David Hsu, Mark Overmars, A. Frank van der Stappen

[8] "Robot Motion and Control 2011" edited by Krzysztof Kozłowski

[9] "Climbing and Walking Robots: Proceedings of the 8th International Conference" by Mohammad Osman Tokhi, G. S. Virk, M. A. Hossain

[10] "Intelligent Autonomous Systems: Foundations and Applications" by Dilip Kumar Pratihar, Lakhmi C. Jain 